\title{
Augmented Assembly using a Mobile Phone
}

\author{
Mika Hakkarainen ${ }^{(1)}$, Charles Woodward ${ }^{(1)}$, Mark Billinghurst ${ }^{(2)}$ \\ VTT Technical Research Centre of Finland ${ }^{(1)}$, The HIT Lab New Zealand, University of Canterbury ${ }^{(2)}$
}

\begin{abstract}
We present a mobile phone based augmented reality (AR) assembly system that enable users to view complex models on their mobile phones. It is based on a client-server architecture, where complex model information is located on a PC, and a mobile phone with the camera is used as a thin client access device to this information. With this system users are able to see an AR view that provides step by step guidance for a real world assembly task. We also present results from a pilot user study evaluating the system, showing that people felt the interface was intuitive and very helpful in supporting the assembly task.
\end{abstract}

\section{INTRODUCTION}

We are interested in exploring how augmented reality (AR) technology can be used to provide intuitive assistance with real world assembly, repair and training tasks. In this paper we present our mobile phone based augmented reality assembly system. We first review previous related work, and then describe our prototype based on a mobile phone client and remote PC server architecture. Finally we provide some preliminary results from a pilot user study evaluating the system.

\section{Related Work}

Several other research groups have explored the use of AR for assisting with real world assembly tasks. One of the earliest of these efforts was augmenting of wire harness assembly at Boeing [1], using a wearable PC with head mounted display attached. More recent augmented assembly and maintenance solutions have been based on portable PC hardware, for example BMW's augmented car maintenance system [3]. Among the first to employ handheld devices were Geiger et al. [2], in an application providing augmented operation instructions for home appliances. Currently hand held (ultra mobile) PCs are a popular choice for portable AR assembly applications, e.g. the ULTRA system [4].

Most recently, mobile phones have been used for AR applications, although not for assembly tasks. The first of these, AR-Phone [5], used Bluetooth to send phone camera images to a remote sever for processing and graphics overlay, taking several seconds per image. Since then, Moehring [7] developed the first example of an AR application running entirely on the phone, while Henrysson ported the ARToolKit library over to the Symbian phone platform [6]. Since that time there have been other examples of mobile phone based AR interfaces, e.g. [8].

As can be seen, AR technology has shown to be useful for

mika.hakkarainen@vtt.fi

charles.woodward@vtt.fi

mark.billinghurst@hitlabnz.org assembly tasks, but until now it has not been delivered on a mobile phone platform. Our work is novel because it provides the first example of an AR assembly interface delivered on a mobile phone, it has unique interface elements that have not been demonstrated before in an AR assembly application, and it also provides a user study of the AR interface.

\section{Demonstration System}

There are a number of challenges in providing a high quality user experience for augmented assembly on mobile phones. Product model formats vary enormously, and the number of polygons can be very large (e.g the 0.5 million triangle model in Figure 1 is very small for industrial applications) which can be difficult to render on the simple graphics hardware of phones. The 3D API on the mobile phones (OpenGL ES) is also limited and the lack of input options for scene graphs requires cumbersome conversions to the mobile phone compatible presentation format.

To overcome these challenges we decided to select a clientserver architecture approach. All of the complex model rendering, handling of model formats and image processing are performed on the PC server, and a still image shows the result delivered to the mobile phone. In this way we can make use of a complex AR rendering system that already exists on the PC environment, and connect it to the mobile phone client with relatively little programming effort. Furthermore, with still images the user can view the augmented content at his or her own pace without having to constantly point the phone towards the target workspace.

Our prototype system is made up of two parts; PC server software and a visualization/controller client on a Symbian (Nokia) smart phone. On the server side we employ a version of the AugAsse software [9]. The tracking system on server side uses a customized version of ARToolkit 4.06, with a set of markers attached to the assembly site. The rendering of the virtual assembly model on the server uses a scene graph and the OpenSceneGraph 2.0 library. Figure 1 shows a pilot application from the AugAsse project.
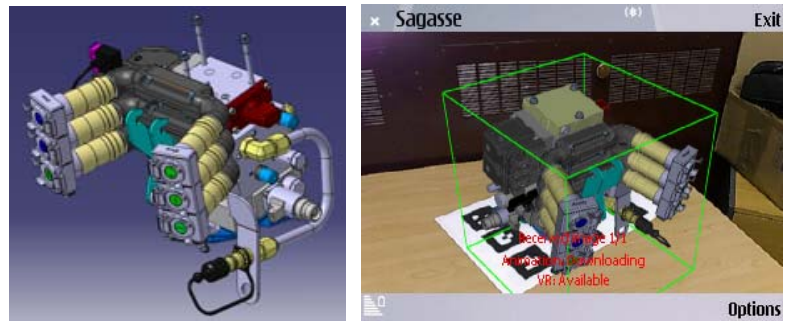

Figure 1 - AugAsse pilot: CAD model and AR view

The phone client software is used to take images of the real assembly site, send them to the server and to show the augmented views to the user on the phone. The server creates a simple animation of the current part being placed in the AR scene by a series of sub images placed on the main image. Each sub image contains a bit map defining which pixels actually show the part and which are transparent. The phone client software is a native Symbian application developed in $\mathrm{C}++$. The client side rendering 
is based on native Symbian bitmaps. Data transfer between the phone and PC uses either Bluetooth (short range) or pure TCP/IP sockets over a wireless LAN connection.

To operate the system, the user takes an image of the assembly with the mobile phone client and sends it to the server. They then uses keypad input to select if s/he wants to see an augmented view of the next step in the assembly task, the previous step, or repeat the current one (from a new view angle). They will then receive the augmented view of the real world. The user places a real model part at the position shown by the AR model, and then takes a new photo to show what the next part and placement should be.

The augmented information is provided as an animated image sequence, properly masked by the real objects and in true perspective (see Figure 2). The phone client also allows the user to zoom the image to take a closer look of the new model and its position in the assembly site. After the user has placed the real (physical) item in place, s/he can move to the next work phase by taking a new image and defining that the current task is now done. All the commands are available as command menu items and the most often used commands are also keypad shortcuts.

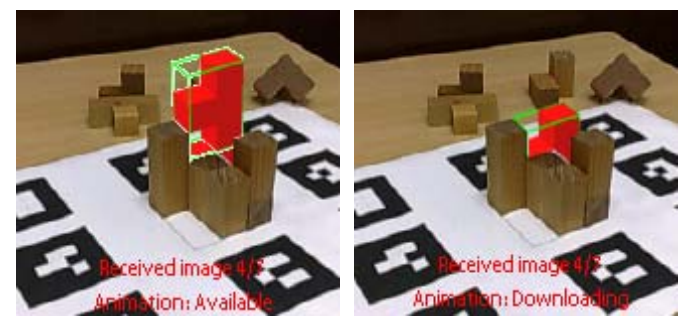

Figure 2 - Augmented assembly of a 3D puzzle on a mobile phone.

\section{Performance}

The client software was implemented with Symbian Series 60 OS and tested on a Nokia N95 phone. The N95 has a 262Mhz Arm 9 processor, graphics hardware, a 5 megapixel camera and a screen resolution of $320 \times 240$ pixels. The current implementation of the system supports sending 640x480 (24 bpp) images from the phone to the server and the server sends back 320x240 (24bpp) images. On the server side the model complexity is the main issue than could cause notable delay in the system performance.

The main bottleneck of the performance is the data transmission times between the phone and PC server. Creating the Bluetooth connection to server takes less than 10 seconds but the bandwidth is less than with WLAN. On the other hand, creating an ad-hoc phone/PC WLAN connection can take up to 60 seconds, but the bandwidth is much higher than with Bluetooth. The average time to send and receive an image was 19.13 seconds over Bluetooth and 3.44 seconds over WLAN, nearly six times faster.

\section{USER EVALUATION}

We conducted a small pilot study to evaluate the effectiveness of the system. For this study we chose an assembly task that involved correctly constructing a wooden 3D soma cube puzzle made up of seven interlocking pieces. When assembled correctly the pieces form a perfect cube. Figure 2 shows an AR view showing how to correctly place one piece of the puzzle.

The system was demonstrated during a local computer science conference. Many people successfully used it to assemble the real puzzle, and from these eight answered a small informal survey. Each of the survey questions was answered on a Likert scale of 1 to 7 where 1 = Not very easy/helpful/enjoyable and 7 = very easy/ helpful/enjoyable. The table opposite shows the average results.

\begin{tabular}{|l|l|}
\hline Question & Avge Result \\
\hline 1: How easy was the system to use? & 4.63 \\
\hline $\begin{array}{l}\text { 2: How helpful was the AR system to } \\
\text { solving the 3D puzzle? }\end{array}$ & 6.50 \\
\hline 3: How easy was it to interact with the system? & 5.38 \\
\hline 4: How enjoyable was it to use the system? & 5.13 \\
\hline
\end{tabular}

The small sample size and lack of conditions to compare against makes it impossible to perform any statistical analysis on these results. However it is clear that the users thought that the system was beneficial, for example in response to Q2, "How helpful was the AR system to solving the 3D puzzle", all of the respondents replied with a 6 or 7 ( 7 = Very Helpful).

In general people enjoyed the application and were very interested in it. Some found it difficult to remember all the buttons but they quickly learned the basic sequences. When told about real life industry case studies of how the technology could be used they found the main concept of the system very useful. The puzzle itself was slightly too "entertaining and simple" to show the real benefit of the system but they enjoyed the puzzle as a "proof of concept" and were interested in how the technology could be applied to real life scenarios.

\section{Conclusions}

This research is the first example of using a mobile phone to provide AR cues for supporting real world assembly tasks. Our AR assembly application uses a thin client on the mobile phone connected to a remote PC server. Images captured by the phone are sent to the server, processed and an AR overlay added before the pictures are sent back for viewing on the phone. Although the typical delay of a few seconds is noticeable, most pilot study users felt that the AR interface was extremely useful in helping them complete a real world assembly task. In the future we plan to explore intuitive techniques, add support for more complex models and to conduct formal user studies.

\section{REFERENCES}

[1] T. Caudell, D. Mizell. Augmented reality - an application of headsup display technology to manual manufacturing process. Proc. 25th Hawaii International Conference on Systems Sciences, Kauii, Hawaii, 1992, Vol 2, pp. 659-669.

[2] C. Geiger, B. Kleinjohann, C. Reimann, D. Stichling. Mobile AR4ALL. In Proc. ISAR'01, New York, USA, Oct 29-30, 2001.

[3] J. Platonov, H. Heibel, P. Meier, B. Grollmann. A mobile markerless AR system for maintenance and repair. Proc. ISMAR'06, Santa Barbara, USA, Oct 22-25, 2006, pp. 105-108.

[4] P. Riess, D. Sticker, G. Voss, W. Mueller-Witting. ULTRA - ultra portable augmented reality for industrial maintenance applications. CG Topics 6/2006.

[5] D. Cutting., M. Assad, A. Hudson. AR phone: Accessible augmented reality in the intelligent environment. In Proc. OZCHI2003, Brisbane, Australia, 2003.

[6] A. Henrysson, M. Ollila. UMAR - Ubiquitous Mobile Augmented Reality. In Proc. MUM2004, Maryland, USA, Oct 27 - 29, 2004.

[7] M. Moehring, C. Lessig, O. Bimber Video See-Through AR on Consumer Cell Phones. Proc. ISMAR'04, Arlington, USA, Nov 2-5, 2004, pp. 252-253.

[8] A. Henrysson, M. Billinghurst, M. Ollila. Face to Face Collaborative AR on Mobile Phones. Proc. ISMAR'05, Vienna, Austria, Oct 5-8, 2005, pp. 80-89.

[9] J. Sääski, T. Salonen, M. Hakkarainen, S. Siltanen, C. Woodward, J. Lempiäinen. Integration of design and assembly using augmented reality. In Proc. IPAS'2008, Chamonix, France, Feb 10-13, 2008. 